\title{
Effects of Acupotomy Treatment on Relapsed Lateral Malleolar Bursitis: A Case Report
}

\author{
Jae Hoon Kim, Jung Hee Lee, Yun-Kyu Lee, Hyun-Jong Lee, Jae Soo Kim \\ Department of Acupuncture \& Moxibustion medicine, College of Korean medicine, Daegu Haany University

\section{재발성 족외과 점액낭염에 대한 도침요법: 증례 보고} \\ 김재훈 ${ }^{(1)} \cdot$ 이정희 $^{(1)} \cdot$ 이윤규 $^{(1)} \cdot$ 이현종 $^{(1)} \cdot$ 김재수 $^{(1)}$ \\ 대구한의대학교 부속 한방병원 침구의학과
}

\begin{abstract}
Objectives: This study details on a case of acupotomy for relapsed lateral malleolar bursitis. Methods: A 71-year-old woman with lateral malleolar bursitis was treated with acupotomy twice in an outpatient setting. A sterilized, disposable, blade width $0.5 \mathrm{~mm}$ $\times$ needle length $50 \mathrm{~mm}$ sized acupotomy was administered on GB40 and BL62 on each visit. Pain intensity assessment using Numeric Rating Scale (NRS) and the response evaluation criteria was conducted right after each treatment. Results: Pain on NRS declined from 5 to less than 1 after twice of acupotomy treatment. This result was regarded as 'Complete Response' using the response evaluation criteria. There were no side effects during whole treatment procedure at all. Conclusions: It is turned out that acupotomy can have a positive clinical effect on lateral malleolar bursitis in this case without any adverse effect. Further controlled studies on acupotomy for lateral malleolar bursitis are required for clinical application.
\end{abstract}

Key words : acupotomy, case report, relapsed lateral malleolar bursitis

\section{Introduction}

A bursa is a type of cyst with a lining of synovial membrane, which can mitigate pressure or friction over certain area. Bursa can trigger inflammation and suppuration under chronic, repetitive pressure ${ }^{1-3)}$.

The lateral malleolar bursa of the ankle is subcutaneous. Sitting on one's own feet, putting on tight shoes, repetitive irritation, excessive pressure, and wound on the feet could result in an inflammatory lesion of lateral malleolar bursa ${ }^{3)}$. The typical conservative treatments for lateral malleolar bursitis are aspiration, anti-inflammatory medication, methylprednisolone acetate injection, OK-432 injection, and so $\mathrm{on}^{3,4}$. However, lateral malleolar bursitis is known to relapse quite frequently, despite these conservative treatments done ${ }^{2)}$. While open excision surgical treatment techniques are also available, post-operative complications including skin necrosis, wound dehiscence, and superficial peroneal nerve palsy could develop ${ }^{5}$. Researchers are continuously devising new treatment techniques, such as ankle arthroscopic bursectomy, in order to prevent these complications from happening ${ }^{5)}$.

\footnotetext{
Received November 18, 2019, Revised December 11, 2019, Accepted December 13, 2019

Corresponding author: Jae Soo Kim

Department of Acupuncture \& Moxibustion Medicine, Daegu Oriental Hospital of Daegu Haany University, 136 Sincheondong-ro, Suseong-gu, Daegu 42158, Korea

Tel: +82-53-770-2108, Fax: +82-53-770-2055, E-mail: jaice@daum.net

CD This is an open access article distributed under the terms of the Creative Commons Attribution Non-Commercial License (http://creativecommons.org/licenses/ by-nc/4.0) which permits unrestricted non-commercial use, distribution, and reproduction in any medium, provided the original work is properly cited.
} 
Studies on minimizing the relapse rate after conservative treatment for lateral malleolar bursitis have recently been reported. For example, one kind of corticosteroid medication, triamcinolone acetonide injection, is emerging as one of the clinically competitive options ${ }^{6}$.

Acupotomy is a new upcoming acupuncture treatment method that requires understanding of both traditional East Asian medicine meridian theory and modern surgical principles simultaneously. Acupotomy induces a soft tissue lesion to recover its own normal kinetic state through peeling and removing abnormally adhered tissues, while balancing yin yang qi blood flow at the same time ${ }^{7}$. Many researchers are trying to prove the advantage of higher-efficacy, quickerrecovery, and the safety of acupotomy treatment compared to ordinary other treatments in musculoskeletal diseases recently ${ }^{8-10)}$.

However, we don't have to confine its clinical, therapeutic capability only to ordinary musculoskeletal diseases. Aspiration, one of the conservative treatments for bursitis must have micro-puncture on target area before to be done. We could also use acupotomy treatment on lateral malleolar bursitis based on its utility against peeling, removing abnormal tissues.

No study has investigated or reported about lateral malleolar bursitis with reference to traditional East Asian medicine principle, and neither have studies about relapsed lateral malleolar bursitis been reported.

In this study, we describe the effect of acupotomy on a patient with lateral malleolar bursitis who did received treatment several times already, but relapse could not be prevented.

\section{Case}

A 71-year-old woman who has swelling at the lateral malleolus of the right ankle as a main complaint, was diagnosed with lateral malleolar bursitis of the right ankle at a local hospital in 2017, but refused surgical recommendation at that moment. Then, she visited the Daegu Hanny university traditional Korean medical hospital. Her symptoms including pain and discomfort of the right ankle had relieved after several conservative treatments, allowing her to maintain her daily life activities comfortably. However, it relapsed after repetitive, hard household labor in June 2019. She was brought to the outpatient department of Daegu Hanny university traditional Korean medical hospital without having received any treatment.

\section{Acupotomy with safety}

Before acupotomy treatment, the practitioner explained the treatment procedure, cautions to be aware of, and probable side-effects after the treatment thoroughly to the patient. The acupotomy treatment began after the informed consent form for case report study was filled by the patient.

A sterilized, disposable acupotomy (DongBang Acupuncture Inc., Republic of Korea; $0.5 \times 50$ mm, whole length 105 $\mathrm{mm}$, length of acupuncture body $75 \mathrm{~mm}$, length of acupuncture head $25 \mathrm{~mm}$, length of cylindrical apex $5 \mathrm{~mm}$, and radius $0.5 \mathrm{~mm}$ ) was used. The patient was in supine position while the acupotomy treatment was done. Acupotomy was performed by Korean medical doctors having department of acupuncture \& moxibustion medicine specialist license (licensed by the Ministry of Health and Welfare, Republic of Korea). The identical practitioner, having more than 20 years of clinical experience, administered the procedures throughout the treatment period. The practitioner first marked the point with a surgical marking pen to make sure at which point the acupotomy treatment would be performed. Sterilization process was precisely administered in order to prevent any minimal adverse effects from happening, for safety in other studies with acupotomy treatment? ${ }^{9}$. The practitioner wore a sterile mask and surgical gloves before treatment. Skin sterilization was done with a disposable alcohol stick swab (Firson Inc., Republic of Korea; Altistick, Ethanol 80\%) and a disposable potadine surgical stick swab (Firson Inc., Republic of Korea; Postick. Povidone Iodine 10\%). Subsequently, a surgical sterilization wrap was draped around the lesion. Acupotomy treatment was performed at the marked point. The practitioner only carried out the 
treatment procedure and did not take part in the evaluation.

Acupotomy was administered on GB40, BL62 by peeling around the swollen point on lateral malleolar bursa. There are no dangerous anatomical structures nearby these points. Aspiration using a $5 \mathrm{cc}$ syringe with a 23-gauge needle (Sungshim Medical., Republic of Korea; Sungshim Insulin Sylinge $0.5 \mathrm{ml} / \mathrm{cc}$ ) followed (Fig. 1). Aspiration was done twice; first through the rear of lateral malleolus to the front, and second through the lower of lateral malleolus to the upper rear end. The practitioner compressed, squeezed lateral malleolar bursa into various directions to aspirate an adequate volume of mucous (Fig. 2).

A circular sterilized bandage was applied on the administration point to prevent secondary infection. After treatment completion, the practitioner checked for any abnormality at the treatment point.

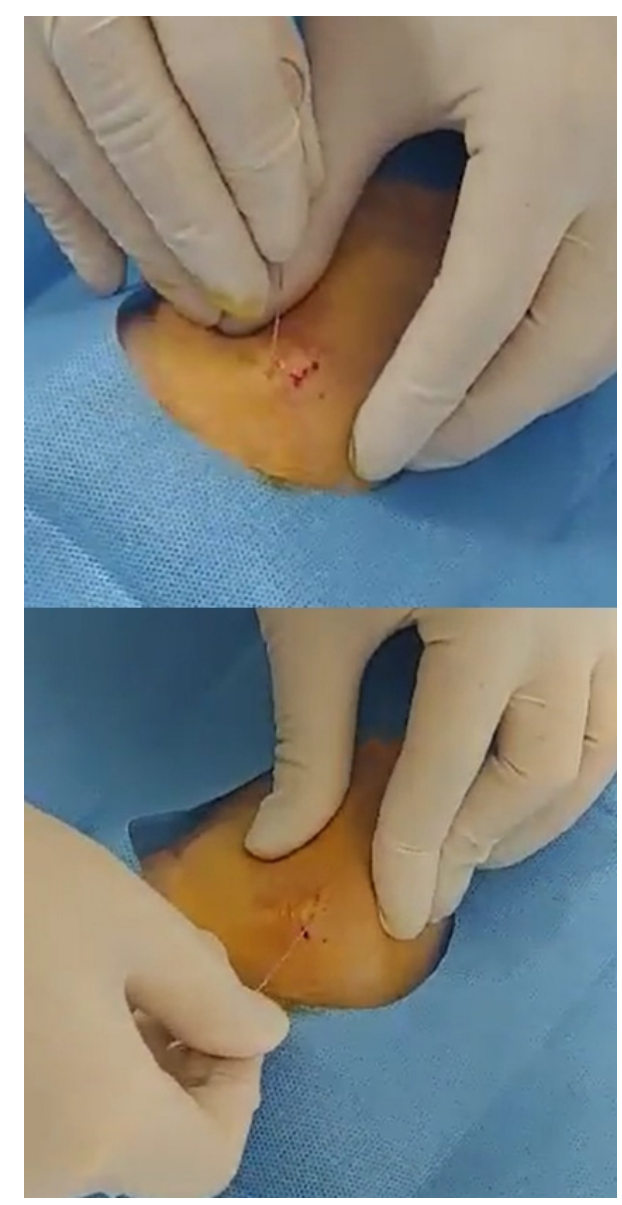

Fig. 1. Application of acupotomy on the lateral malleolus.
The status of patient was evaluated just before treatment at each outpatient visit by identical assessor. The symptoms and size of lateral malleolar bursitis were evaluated and measured at each outpatient visit. The clinical results were assessed by response evaluation criteria that were modified from the guidelines of the response to treatment for a solid tumor $^{4,11)}$. These response evaluation criteria include the state of fluctuation, shrinkage of the bursa, and swelling of the soft tissue, scored as 'Complete Response', 'Partial Response', and 'No Response' (Table 1).

Numeric Rating Scale (NRS) score is also used to assess the severity of pain or symptom. On a scale of $0 \sim 10$, where 0 indicates "no pain at all" and 10 indicates "the most severe pain imaginable", the patient was asked to say a number index that describes the symptom best in a relaxed position ${ }^{12}$.

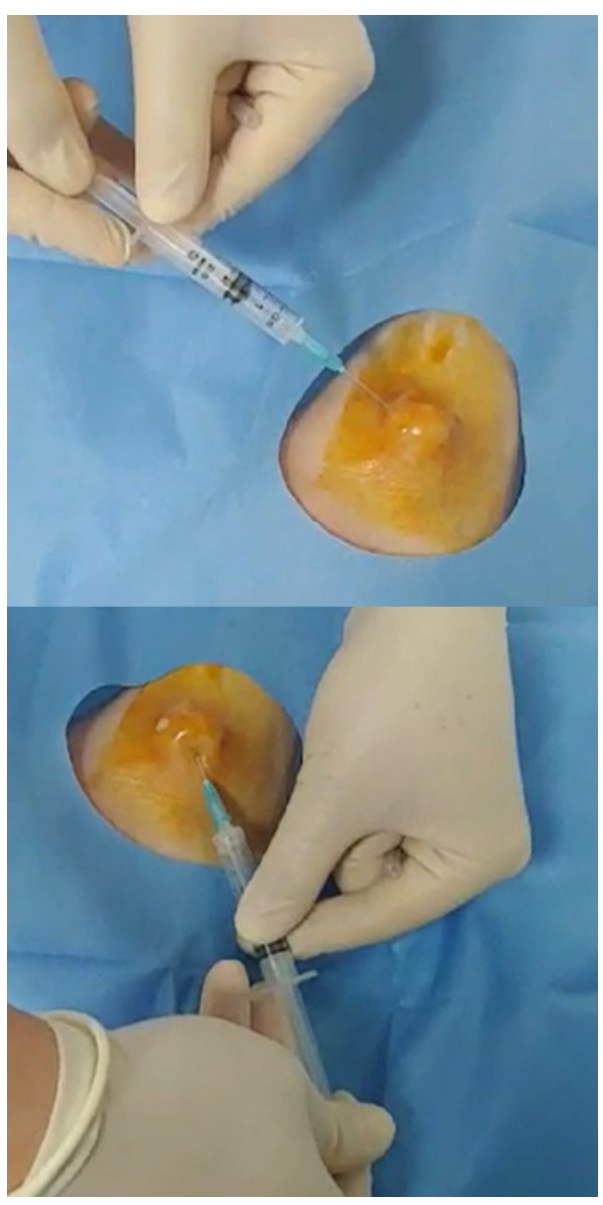

Fig. 2. Aspiration of the lateral malleolar bursa after application of acupotomy. 


\section{Progress}

A distinct swelling on lateral malleolar bursa of right ankle was observed at the first visit on 2019.07.04 (Fig. 3). NRS score was assessed as 5. Pain on the lateral malleolus of right ankle was exacerbated the previous night. Pain was most severe during walking with shoes on. Aspiration was performed just after acupotomy treatment. Approximately 3 cc of ivory-white mucous was drawn out.

On visit 2, the swelling on the lateral malleolus of the right ankle became quite small after being treated only once with acupotomy; the right ankle seems no different from the left ankle at first sight (Fig. 4). NRS score was assessed as 1 on

Table 1. Response Evaluation Criteria for Malleolar Bursitis of the Ankle)

\begin{tabular}{lccc}
\hline $\begin{array}{c}\text { Response to } \\
\text { treatment }\end{array}$ & Fluctuation & Shrinkage & $\begin{array}{c}\text { Soft tissue } \\
\text { swelling }\end{array}$ \\
\hline 'Complete Response' & Complete & No & None or minimal \\
'Partial Response' & Partial & Yes & Yes \\
'No Response' & No change & Yes & Yes \\
\hline
\end{tabular}

this visit. There was no exacerbation of pain after the previous treatment. Acupotomy treatment with aspiration was performed. The treatment method was identical to the previous one. Ivory-white mucous was drawn out as in last visit, but the volume of mucous was less than $0.5 \mathrm{cc}$.

The patient did not visit after second treatment. We conducted a telephonic follow-up about the symptom on 29 July 2019. The patient did not come because the symptom became too weak, thinking that there was no need to get other treatments. Pain on her lateral malleolus of right ankle did increase while walking and putting on shoes slightly. However, it was so relieved that it did not become bothersome during her daily life. NRS score was assessed as $0-1$, which means lesser than 1 . The total NRS progress is described as a graph (Fig. 5).

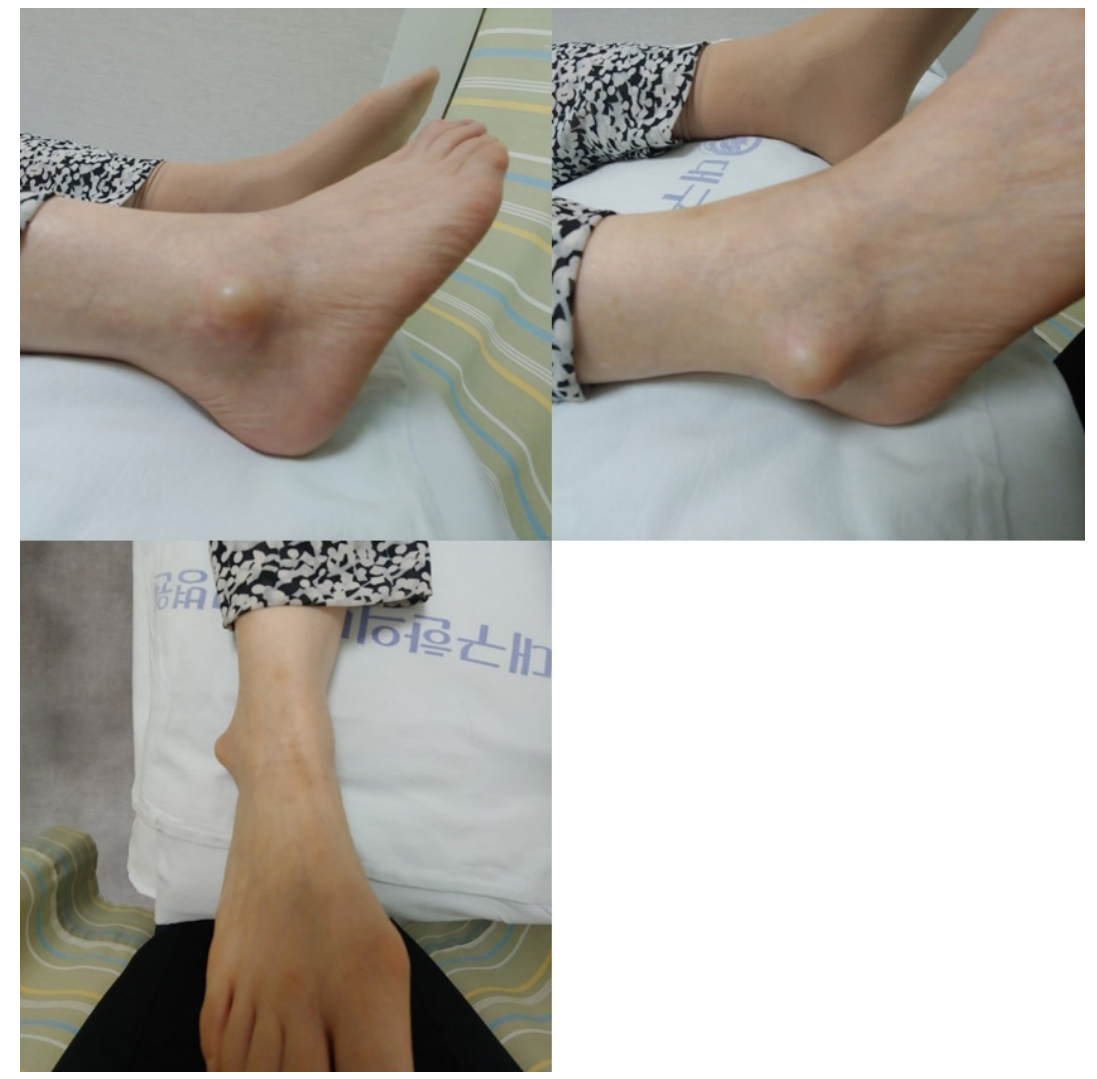

Fig. 3. Picture of the lateral malleolus of the right foot on Visit 1. 


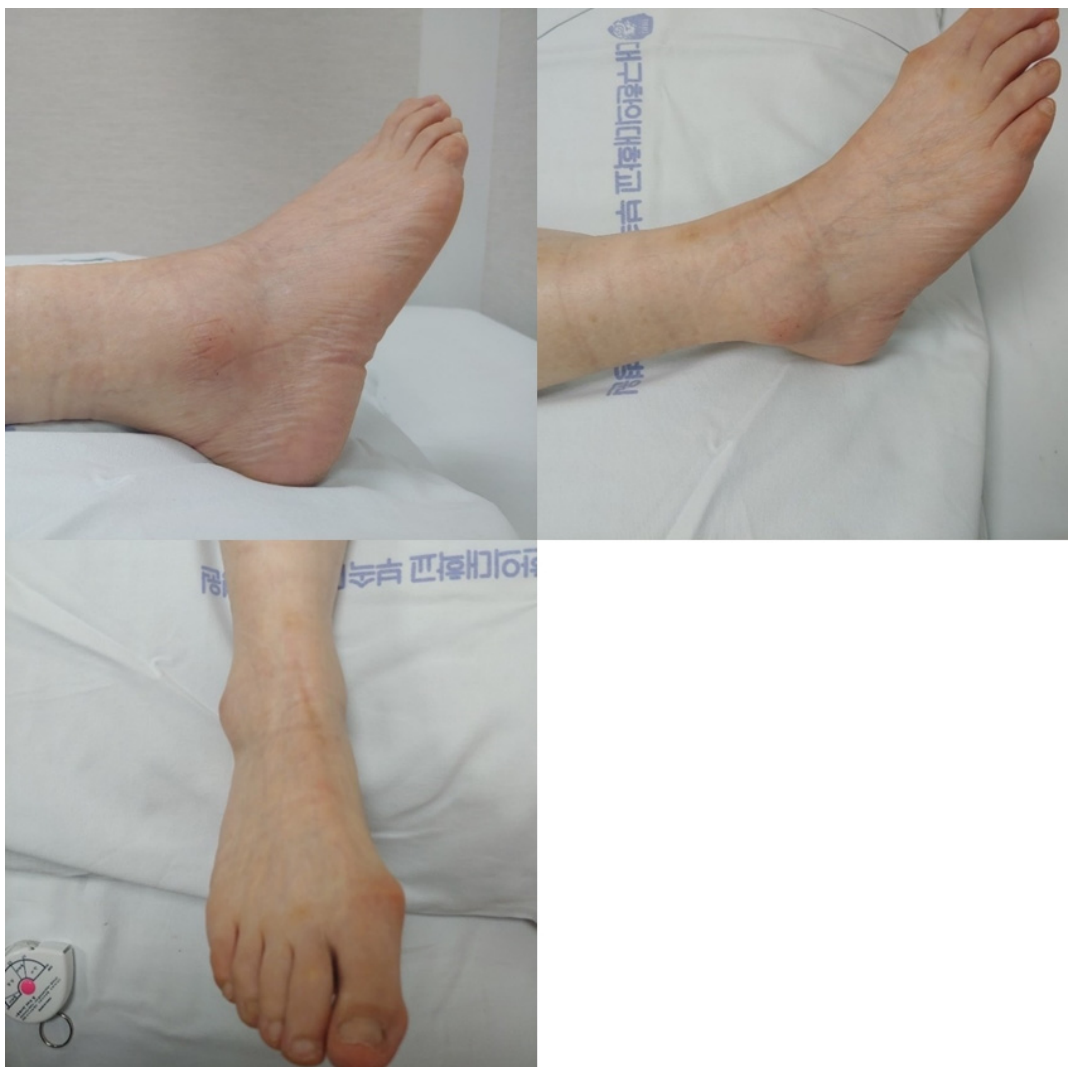

Fig. 4. Picture of the lateral malleolus of the right foot on Visit 2.

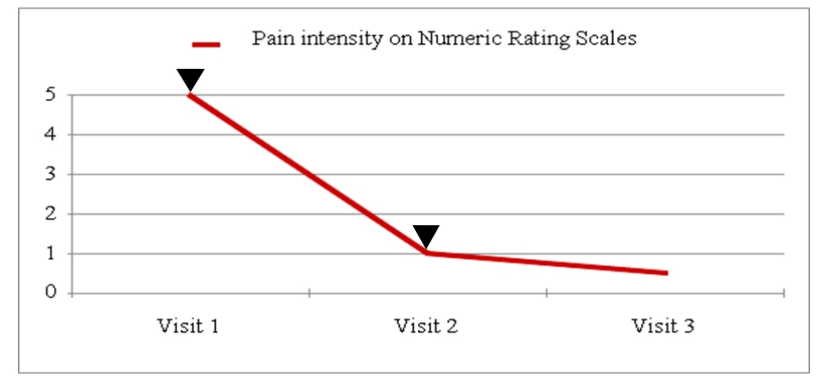

Fig. 5. Changes of pain intensities on Numeric Rating Scales. Acupotomy was administered on Visit 1, Visit 2.

\section{Discussion}

In this study, it is turned out that patient's symptoms were improved after a single acupotomy treatment. NRS scores declined from 5 at first to 1 , declined more at after visits. Assessing the NRS changes, patient's symptoms maintained an improved state. Based on the response evaluation criteria from Table 1, a state of 'Complete Response' appears to be well maintained. There has not been adequate pathological research regarding the factors that affect relapses or the latent period until relapse; it is not difficult to acknowledge that acupotomy treatment is effective, as demonstrated.

There are 2 types of bursa. One is the anatomical bursa, which develops by moderate friction caused by ordinary movement between tendons and adjacent structures. The adventitious bursa is the other one. It is primarily developed by localized, repetitive, abnormal pressure or friction on a specific part ${ }^{1-3}$. It also has a thicker fibrous wall compared with an ordinary bursa, increasing the possibility of inflammatory changes. Therefore, bursitis is more likely to affect an adventitious bursa than any other type of bursa ${ }^{13)}$.

In the case of moderate lateral malleolar bursitis, subjective symptoms are often mild. Therefore, ordinary conservative treatments, such as aspiration, applying a compression bandage, corticosteroid injections, or orthopedic posture correction are initially recommended ${ }^{5,14}$. The relapse rate of lateral malleolar bursitis is quite high, though the time after conservative treatment until relapse may differ 
among patients. The relapse rate reported after orthopedic posture correction was about $43.7 \%$, and after aspiration, or applying a compression bandage was about $35.0 \%$, according to previous studies ${ }^{3,5}$.

Patients who repeatedly wear hard shoes, like skaters, or have the habit of sitting on their feet under the buttocks create chronic excessive pressure on the bursa ${ }^{3}$. Cases of bursitis associated with chronic excessive pressure could progress from bursitis to suppurative inflammation, affecting nearby joints as well ${ }^{6}$. Therefore, some researchers have suggested that symptoms such as redness, hyperesthesia, enlarged lymph nodes, and heat sensation should be monitored in patients who have lateral malleolar bursitis ${ }^{11}$.

Surgical treatment is recommended when conservative treatment is not effective. Initially, post-operative complications after open excisional surgery were reported to include skin necrosis, wound dehiscence, and superficial peroneal nerve palsy, ${ }^{5,6}$. New surgical techniques have reported for minimized post-operative complications. Arthroscopic ankle bursectomy, introduced to minimize post-operative complications, might be challenging when deciding the excision range for infected tissues having fistulas associated with post-operative wound infections ${ }^{5}$. This procedure is also more technically difficult to perform compared to other surgical techniques ${ }^{14}$. The lateral calcaneal artery adipofascial flap, another surgical treatment technique, was also reported to be used for treating malleolar bursitis ${ }^{15}$. This technique, however, requires quite a large incision. Surgeons also must be well versed in the patient's local anatomical ankle structure, such as blood vessel patency and blood flow variance prior to using this surgical technique. Due to these limitations, these surgical techniques could not be universally performed, though no better alternative techniques exist ${ }^{14,15}$.

A previous study reported that triamcinolone acetonide injections are simple, have a lower relapse rate, have fewer complications, and are a highly effective conservative treatment ${ }^{\text {? }}$. Another study recommended surgical treatment over conservative treatment and discussed the results of methylprednisolone acetate injections after aspiration of the bursa, demonstrating that this treatment did not prevent relapse of bursitis ${ }^{3)}$. Other study suggested that localized corticosteroid injections should be limited to a maximum of twice, throughout the whole treatment period ${ }^{16}$. The conclusions of studies focusing on corticosteroid injections as a conservative treatment method could not reach a consensus regarding the methodology. Each study used different kinds of corticosteroids and different volumes for injection, creating variability in the results analyses. Corticosteroid injections could be indeed effective to treat bursitis. However, those controversies and limitations should be solved to get universal utility.

Acupotomy evolved from 'Chinese small knife acupuncture therapy' in 1976, combining traditional Chinese medicine meridian theory and modern surgical principles ${ }^{17}$. From a pathological point of view, inflammatory fluid effusion within the fascia or bursa could increase internal pressure on soft tissues, leading to abnormal localized excessive stress. This stress may create subtle circulatory disturbances nearby, causing pain in the soft tissues. At this point, 'the peeling off method' of acupotomy can be utilized for lesion treatment. In a bursa with abnormal effusion, cutting the capsule and peeling it off could be useful to decrease the abnormally increased internal pressure and remove the inflammatory fluid. This treatment may also be administered on the fascia, joint capsules and cortical bone, peeling around the stiffened ligaments nearby. This method could lower internal pressure on the soft tissues, improve the nearby circulation, and promote healing of the inflammatory lesions ${ }^{18}$.

Acupotomy of lateral malleolar bursitis might be comparatively rapider and easier to perform than any other procedures previously discussed ${ }^{8)}$. Many clinical studies have variously shown the remarkable effect of acupotomy treatment in reducing inflammation, elevating pain threshold, relieving pain, regulating the inflammatory factors (such as interleukin- $1 \beta$, inteleukin- 6 and tumor necrosis factor- $\alpha$ ), lowering nitric oxide and so on ${ }^{10)}$. Some studies suggest that acupotomy treatment has higher, longer lasting cure effect than any other treatments including nerve block or other surgical methods ${ }^{10)}$. Though acupotomy treatment may have higher risk of adverse effect than using conventional acupuncture 
technique, few mild adverse effects were reported such as erythema or ecchymosis ${ }^{9-10)}$. There were no serious or severe adverse effect reported after acupotomy treatment ${ }^{9)}$.

There might be lesser need to think about post-operative recovery period or risk of cutaneous complications after acupotomy treatment, making itself as a much-safer procedure than any other treatment methods. We could also suggest that acupotomy has the advantage of a quicker recovery and a more effective analgesic effect compared to other treatment methods. It showed long-lasting effects and fewer complications after treatment. Therefore, acupotomy might promote healing at the lesion that could lead to a near 'fully recovered' state ${ }^{18)}$.

However, in this study, no radiological imaging was used to determine the severity of the patient's symptoms or the level of lesion improvement after treatment. Additionally, the number of patients was too limited to obtain meaningful results. The evaluation criteria were not sensitive enough to monitor changes during the treatment period. We could not evaluate the effects of acupotomy treatment independently during this study, as it did not include conventional treatment cases as controls to compare the results. Therefore, there were limitations when assessing the objective, accurate, and meaningful effects of acupotomy treatment.

In this study, we explored the possibility to utilize acupotomy treatments for lateral malleolar bursitis, a rare musculoskeletal disorder. The results suggest that the effectiveness of acupotomy treatment may be comparable to those of other conventional treatments.

Further studies including case-series or observational studies which could exclude those limitations mentioned above and include meaningful evidence should be pursued to evaluate the safety and efficacy of acupotomy treatment in a broader target area. This method could emerge as a new solution for other difficult-to-treat diseases.

\section{Acknowledgement}

The authors received no specific funding for this work.

\section{Funding}

None.

\section{Data availability}

The authors can provide upon reasonable request.

\section{Conflicts of interest}

The authors have declared that no conflicts of interest exists.

\section{References}

1. Hernandez PA., Hernandez WA., Hernandez A. Clinical aspects of bursae and tendon sheaths of the foot. J Am Podiatr Med Assoc. 1991 ; 81(7) : 366-372. doi: 10.7547/87507315-81-7-366

2. Ruangchaijatuporn T., Gaetke-Udager K., Jacobson J. A., Yablon C. M., Morag Y. Ultrasound evaluation of bursae: anatomy and pathological appearances. Skeletal Radiol. 2017 ; 46(4) : 445-462. doi: 10.1007/s00256-017-2577-x

3. Avci S, Şayli U. Lateral premalleolar bursitis as a result of sitting on the foot. Foot Ankle Int. 2001 ; 22 : 64-66. doi: 10.1007/ s00256-017-2577-x

4. Park KH, Lee J, Choi WJ, Lee JW. OK-432 sclerotherapy for malleolar bursitis of the ankle. Foot Ankle Int. 2013 ; 34(10) : 13891394. doi: $10.1177 / 1071100713488091$

5. Choi JH, Lee KT, Lee YK, Kim DH, Kim JR, Chung WC, et al. Endoscopic versus open bursectomy of lateral malleolar bursitis. Knee Surg Sports Traumatol Arthrosc. 2012 ; 20(6) : 1205-1208. doi: 10.1007/s00167-011-1581-2

6. Woo SH, Kim JS, Son SM, Shin WC. Triamcinolone acetonide injections for lateral malleolar bursitis of the ankle. J Korean Foot Ankle Soc. 2019 ; 23(1) : 12-17. [In Korean] doi: 10.14193/jkfas. 2019.23.1.12

7. Yuk DI, Kim KM, Jeon JH, Kim YI, Kim JH. A review of trends for 
acupotomy. The Acupunct. 2014 ; 31(3) : 35-43. [In Korean] doi: 10.13045/acupunct. 2014041

8. Jun SA, Park MS, Oh SJ, Lee JH, Gong HM, Choi SH, et al. Efficacy and safety of miniscalpel acupuncture in knee degenerative osteoarthritis patients: a study protocol for a randomized controlled pilot trial. Korean J Acupunct. 2016 ; 33(2) : 67-74. doi: 10.14406/acu.2016.010

9. Yoon SH, Kwon CY, Leem J. Adverse events of miniscalpel-needle treatment in Korea: A systematic review. European Journal of Integrative Medicine. 2019; 27 : 7-17. doi: 10.1016/j.eujim.2019. 02.002

10. Kwon CY, Yoon SH, Lee B. Clinical effectiveness and safety of acupotomy: An overview of systematic reviews. Complementary Therapies in Clinical Practice. 2019; 36 : 142-152. doi: 10.1016/ j.ctcp.2019.07.002

11. Therasse P, Arbuck SG, Eisenhauer EA, Wanders J, Kaplan RS, Rubinstein L, et al. New guidelines to evaluate the response to treatment in solid tumors. J Natl Cancer Inst. 2000 ; 92(3) : 205-216. doi: 10.1093/jnci/92.3.205

12. Lee HJ, Lee SS, Lee YH. The study of pain and functional disability scales for low back pain. J Korean Acupunct Moxib Soc.
$2002 ; 19(2): 137-148$. [In Korean] (no doi)

13. Treg DB, Thomas EV, Lyle JM. Malleolar bursitis in figure skaters: indications for operative and nonoperative treatment. AmJ Sports Med. 2000 ; 28(1) : 109-111. doi: 10.1177/03635465000280010301

14. Jang HS, Choi HJ. Surgery for synovial fistula after excision of the lateral malleolar bursitis of the ankle: a case report. J Korean Foot Ankle Soc. 2015 ; 19(3) : 118-121. [In Korean] doi: 10. 14193/jkfas.2015.19.3.11

15. Hashimoto I, Yoshinaga R, Toda M, Nakanishi H. Intractable malleolar bursitis treated with lateral calcaneal artery adipofascial flap. Br J Plast Surg. 2003 ; 56(7) : 701-703. doi: 10.1016/ S0007-1226(03)00363-1

16. Nichols AW. Complications associated with the use of corticosteroids in the treatment of athletic injuries. Clin J Sport Med. 2005 ; 15(5) : 370-375. doi: 10.1097/01.jsm.0000179233.17885.18

17. Korean Acupuncture \& Moxibustion Medicine Society. The Acupuncture and Moxibusition Medicine. 4th edition. Seoul (Korea): Jipmoon. 2012; 193

18. Kang CS, Yang HM. Acupotomy and Clinic. Seoul (Korea): DAU. $2011 ; 98-112$ 